\title{
Dietary patterns in older British men: the influence of early life social circumstances and area of residence
}

\author{
J. L. Atkins ${ }^{1}$, P. H. Whincup ${ }^{2}$, R. W. Morris ${ }^{1}$ and S. G. Wannamethee ${ }^{1}$ \\ ${ }^{1}$ Department of Primary Care and Population Health, University College London, London, NW3 $2 P F$ and ${ }^{2}$ Population \\ Health Research Centre, Division of Population Health Sciences and Education, St George's, University of London, London, \\ SW17 ORE, UK
}

Diet quality is a major modifiable determinant of morbidity and may be particularly important in the elderly, who are at high risk of chronic disease ${ }^{(1)}$. However, few studies have examined factors which influence diet quality in older adults in Great Britain ${ }^{(2)}$. The aim of the present study was to examine the influence of area of residence and early life social circumstances on dietary patterns among older British men.

Cross-sectional data from 4252 older men (aged 60-79 years) from the British Regional Heart Study were analysed. Food frequency questionnaire data were used to examine daily fruit and vegetable consumption and to generate two dietary scores - the Healthy Diet Indicator (HDI), based on the World Health Organization's dietary guidelines for intake of nutrients and food components ${ }^{(3)}$, and the Elderly Dietary Index (EDI), based on the frequency of consumption of specific foods and food groups ${ }^{(4)}$. Higher scores of both the HDI and the EDI indicated greater compliance with dietary recommendations. Linear and logistic regression analyses assessed associations of adult and childhood social class (based on father's occupation) and area of residence with dietary patterns, adjusting for age, energy intake, cigarette smoking, physical activity and body mass index.

The mean HDI score was 3.0 (range: 0-7) and the mean EDI score was 26.7 (range 14-35). Only 19.2\% of participants consumed fresh fruit and vegetables daily. EDI and HDI scores were modestly correlated $(r=0.24, p<0.001)$. Men with non-manual childhood social class had higher EDI scores (adjusted mean difference $=0.54, p<0.001$ ) and were slightly more likely to consume fruit and vegetables daily (OR: 1.22, 95\% CI: 0.98-1.51), independent of adult social class. Men with non-manual adult social class also had higher EDI scores (adjusted mean difference $=1.30, p<0.001)$ and were much more likely to consume fruit and vegetables daily $(\mathrm{OR}$ : $1.56,95 \% \mathrm{CI}$ : 1.26-1.93), independent of childhood social class. The HDI score was not associated with childhood or adult social class. Region of birth and region of residence were related to daily fruit and vegetable consumption and to both the HDI and EDI scores, with the highest consumption/scores seen in the Southern region and the lowest in Scotland.

In conclusion, dietary patterns in older men are influenced by area of residence and social class, with adult social class being more influential than early life social class in determining dietary patterns.

1. World Health Organization. (2003) WHO Technical Report Series No 916.

2. Maynard M, Gunnell D, Ness AR, Abraham L, Bates CJ, Blane D (2006) Eur J Public Health 16(3), 316-24.

3. Huijbregts P, Feskens E, Rasanen L, Fidanza F, Nissinen A, Menotti A et al. (1997) BMJ 315(7099), $13-7$.

4. Kourlaba G, Polychronopoulos E, Zampelas A, Lionis C, Panagiotakos DB (2009) J Am Diet Asocs 109(6), 1022-30. 